\title{
MedienPädagogik
}

Zeitschrift für Theorie und Praxis der Medienbildung

\section{Betreutes Monitoring in einer prozessbegleitenden pädagogischen Evaluation (Projekt WiBA-Net)}

\author{
Jennifer Mockenhaupt und Thomas Trebing
}

\section{Einleitung: Betreutes Monitoring als Evaluations- und Forschungsmethode}

Der folgende Text behandelt den Einsatz des betreuten Monitoring als empirischer, medienpädagogischer Methode im Rahmen von Online-Lehre und Blended Learning. ${ }^{1}$ Das betreute Monitoring besteht aus einer computer- und internetunterstützten Datenerhebung, die den realen Prozessen und Kontexten vor Ort, z.B. an der Universität, gegengelesen und den Beteiligten zurückgemeldet wird. Die Methode expliziert der Text exemplarisch anhand des interdisziplinären, universitären Blended Learning BMBF-Projektes WiBA-Net ${ }^{2}$. WiBA-Net umfasst die multimediale Aufbereitung des Lehrstoffes für die Fächer Baustofflehre und Werkstoffmechanik in der Aus- und Weiterbildung von Bauingenieuren und Architekten. Das Lehr- und Lernnetz entwickelten arbeitsteilig Bauingenieure, Architekten, Informatiker, Pädagogen und Webdesigner aus sechs Universitäten. Die Pädagogik hatte dabei eine von 16 Stellen inne.

Das betreute Monitoring wurde als Methode im Rahmen eines Methodenmixes (Triangulation) der prozessbegleitenden Evaluation eingesetzt, die zunächst kurz skizziert wird. Die Methode des betreuten Monitorings beschreibt der Text danach anhand eines Erfahrungsberichtes. Der Erfahrungsbericht umfasst die Konzeption, die Durchführung und die praktische Arbeit mit dem Monitoring in einem Semester im interdisziplinären Projekt WiBA-Net. Den Abschluss bildet eine Forschungsfrage zum angemessenen Seitenumfang von Online-Kursen, für deren Beantwortung das betreute Monitoring Hinweise liefert.

1 Wenn die Online-Lehre die traditionelle Präsenzlehre nur ergänzt, sprechen wir aus der Rezipientenperspektive von Angereichertem Lernen oder Augmented Learning. Wenn die Online- Lehre möglichst viele wesentliche Elemente und Prozesse zumindest auch bereitstellt, dann nennen wir das Lernen Blended Learning. Sind wesentliche Elemente und Prozesse ausschließlich online zugänglich bzw. möglich, sprechen wir von Onlineoder Telemedialem Lernen.

2 WiBA-Net $=$ Bundesweites multimediales Lehr- und Lernnetzwerk für das Fach Werkstoffe im Bauwesen für Bauingenieure und Architekten. Laufzeit 2001 - 2003. Vgl. www.wiba-net.de 


\section{Pädagogische Aufgaben im Projekt WIBA-Net}

Im Laufe des Projekts haben ein Professor, zwei wissenschaftliche und zwei studentische Mitarbeiter im pädagogischen Team in verschiedenen Umfängen mitgewirkt. Das pädagogische Team des Arbeitsbereiches „Bildung und Technik“ des Institutes für Allgemeine Pädagogik und Berufspädagogik der Technischen Universität Darmstadt übernahm im Projekt WiBA-Net folgende Aufgaben:

- Umgebungsanalyse des realen Studienalltags des Faches WiBA (z.B. Vorlesung, Übung, Lernorganisation) und der ihn beeinflussenden Größen (z.B. Aufwand für andere Fächer) zu Projektbeginn. Die Rückmeldung der Ergebnisse sollte den Projektpartnern Hinweise geben, wie das Lernnetzwerk nachhaltig in den realen Lehrund Studienalltag eingefügt werden kann.

- Pädagogische Beratung der Autoren und Techniker zu Vermittlungs- und Gestaltungsfragen.

- Prozessbegleitende pädagogische Evaluation von einzelnen Lehrpfaden mit Studierenden und des Online-Lernnetzes insgesamt mit Studierenden und Lehrenden.

- Redigierung von Lehrpfaden nach pädagogischen Gesichtspunkten. Die pädagogischen Experten nahmen die Sicht von „potenziellen Lernenden“ ein und arbeiteten als Fachleute für Vermittlung unter lernerbezogenen Gesichtspunkten von den Autoren ausgewählte Lehrpfade durch.

Der folgende kurze Abschnitt behandelt die Aufgabe der pädagogischen Evaluation, in deren Rahmen das betreute Monitoring zum Einsatz kam.

\section{Prozessbegleitende pädagogische Evaluation}

Die Evaluation war pädagogisch, weil sie sich am Ideal des mündigen Bauingenieurs bzw. Architekten orientierte. Im Mittelpunkt standen die nutzenden Lernenden mit ihren Bedürfnissen als Lernende. Die pädagogische Evaluation verstand sich als Anwalt der Nutzenden. Nutzende waren vordringlich die Studierenden des Bauingenieurwesens und der Architektur, aber auch Lehrende, Mitarbeiter und Autoren der Lehrpfade. Unmittelbares Ziel der pädagogischen Evaluation war die nachhaltige Nutzung des OnlineLernnetzwerks. Die nachhaltige Nutzung sollte durch die Selbstbestimmung und durch größere Entscheidungsmöglichkeiten gefördert werden, wie mit dem System umgegangen werden kann. Für das System bedeutet das z.B. die Flexibilität oder Adaptierbarkeit und für die Inhaltsaufbereitung die Handlungs- bzw. Problemorientierung. Diese Orientierung kann sich direkt in der sachlichen Anregung bzw. Forderung nach Problemorientierung bei der Stoffaufbereitung ausdrücken, nach dem notwendigen Einbinden von (Selbst-)Tests mit anregend differenzierendem Feedback oder der Orientierung an Aufgaben und Prozessen aus der beruflichen Praxis. Das selbstgesteuerte Lernen und seine Nachhaltigkeit sollen 
aber besonders verschiedene Integrationsmöglichkeiten für die Lernenden in ihr Studium fördern, d.h. realistisch mögliche und geförderte verschiedene Lerngründe, -orte, -Zeiten, -wege, sowohl im System als auch im realen Alltag.

\subsection{Integration in den Alltag: Reale Lernumgebung, Relevanz und Mehrwert}

Als wichtigste Größe oder Bedingung, die für die nachhaltige Nutzung zu erfüllen sei, erwies sich die Akzeptanz und Integration des Lernsystems in den Alltag der nutzenden Lernenden und Lehrenden. Andere Größen wie die Vollständigkeit, die besonders die Bauingenieure als wichtigste Nutzungsbedingung ansahen, haben sich als weniger bedeutsam gezeigt: Auch das jeweils Vorhandene kann, wenn es von den Lehrenden passend eingesetzt wird, die Integration und damit die Nachhaltigkeit bei Lehrenden und Lernenden ermöglichen bzw. fördern.

Integration und realer Alltagsbezug sind nur unter mindestens drei Bedingungen möglich:

- Im Blick muss die reale Lernumgebung (der Studierenden ${ }^{3}$ ) sein, nicht nur eine technisch reduzierte Lernumgebung, wie sie das Online-System selbst darstellt. Wege, Orte, Zeiten, Sozialformen des Lernens und der Stellenwert eines Faches im Verhältnis zu anderen Fächern fördern oder behindern Integration und Alltagsbezug. Sie müssen berücksichtigt, d.h. erfasst und ausgewertet werden. ${ }^{4}$

- Die Relevanz, warum und wie das System den Nutzenden nützt, muss für die Lerner transparent sein: Warum muss ich das lernen? Warum ist das wichtig? Was habe ich davon für meinen Lernprozess? Nur wenn dieser Sinn des Lernens für die Nutzenden klar ist, können Lernprozesse angestoßen werden.

- Das Lernsystem muss den Nutzenden „etwas bringen“, d.h. einen deutlichen Mehrwert gegenüber herkömmlichen Lernmitteln wie Vorlesung, Skript, Buch oder Übungsklausur für sie darstellen, der auch nutzbar ist. Vorteile können für die Nutzenden sein: alternativer Zugang zum Stoff, knappere Inhalte, besser strukturierte Inhalte, andere, aktivierendem Präsentationsformen als Text (Bilder, Tabellen, Animationen, Simulationen), Übungsmöglichkeiten wie Selbsttests, schneller Zugriff von zu Hause, statt über die Bibliothek, effektivere Prüfungsvorbereitung usf.

Für die Nachhaltigkeit der Nutzung, für die angestrebte Akzeptanz bei den Nutzenden und bei der Integration in den Alltag spielten die reale Lernumgebung, die real vorhandene und transparente Relevanz und der Mehrwert eine entscheidende Rolle.

3 Analoges gilt für die reale Lehrumgebung der Lehrenden.

4 In der Literatur heißt dieser Aspekt „Umfeldanalyse“ (Blumstengel 1998. Im Hypertext „Bedarfsanalyse“ anklicken). Wir nennen ihn „Umgebungsanalyse“, um ihn von dem engeren technischen Begriff der „Anforderungsanalyse“ abzugrenzen. 


\subsection{Eingesetzte Methoden}

Die prozessbegleitende Evaluation bediente sich insgesamt folgender Methoden der empirischen Sozialforschung:

- Dokumentanalyse:

- Projektantrag (Zielgruppen, technische und organisatorische Rahmenbedingungen usf.), im Projekt erstellte Papiere.

- Experiment:

- Lehrpfadcheck: Lernen mit einem Lehrpfad in einer Laborsituation mit anschließender Befragung mittels Leitfadeninterview.

- Befragungen:

- Lehrpfadcheck: Qualitative Leitfadeninterviews (face-to-face).

- Reale Nutzung Studierende: ${ }^{5}$ Kurze qualitative Leitfadeninterviews (telefonisch) mit quantitativen Elementen.

- Reale Nutzung Lehrende: Kurze qualitative Leitfadeninterviews (telefonisch) mit quantitativen Elementen.

- Feedback nach den Redigierungen von den Autoren: Kurzfragebogen per EMail.

- Beobachtungen:

- Nicht teilnehmende Beobachtung des Lernverhaltens am Rechner mit anschließendem Videoprotokoll des Bildschirms beim Laborlernen.

- Teilnehmende Beobachtung der Kommunikation im Shared Workspace BSCW ${ }^{6}$ mit den Projektpartnern und in Chat und Forum des Systems mit Projektpartnern und Studierenden.

- Teilnehmende Beobachtung der Kommunikation bei den Projekttreffen und in Arbeitsgruppen.

- Nicht teilnehmende Beobachtung der realen Nutzung durch die Studierenden im Betreuten Monitoring. Beobachtet wurden die studentischen Anmeldungen (Logins) und Kursnutzungen im Online-System.

Die Ergebnisse dieser Erhebungen wurden zeitnah ausgewertet ${ }^{7}$ und sofort für alle Beteiligten veröffentlicht ${ }^{8}$, in Vorträgen bei Projekttreffen und Texten im

5 Zum theoretischen Hintergrund vgl. den Vortragstext von Sesink (2002, S. 4): „Im genannten Projekt hatten wir nun (und haben wir noch) den Weg zu gehen von der bildungs- und medienpädagogischen Theorie zur Entwicklung einer in den realen Studienalltag integrierbaren Lernumgebung.“

6 BSCW = Basic Support for Cooperative Work. Vgl. zum System BSCW [http://bscw.gmd.de].

7 Die Interviews mit den studentischen Lernexperten wurden nur bis zur Stufe des thematischen Vergleichs ausgewertet, verschiedene Interviews, Kategorien wurden aus dem Fragenkatalog und einem Interview gewonnen und in allen Interviews geprüft. Die Stufen der Sozialwissenschaftlichen Konzeptualisierung und theoretischen Generalisierung haben wir nicht erreicht, unter anderem deshalb, weil wir mit den generierten Kategorien ausreichend (und ressourcenorientiert) die gewünschten Ergebnisse sicherstellen und ins Projekt zurückmelden konnten. Vgl. Meuser/Nagel (1991, S. 441-471).

8 Mayring etwa spricht von der Dokumentation des Forschungsprozesses, um ihn nachvollziehbar (transparent) zu machen. Vgl. Mayring (2002, S. 140-148, hier S. 144). 
Shared Workspace BSCW ${ }^{9}$ Die Ergebnisse der Evaluation sind selbstverständlich in die anderen Aufgaben eingeflossen ${ }^{10}$, ebenso wie die anderen Aufgaben Hypothesen und Kategorien für die Evaluation erzeugt haben (z.B. „Didaktischer Mehrwert“ und „Angst vor Zusatzbelastung“ aus unserer Umgebungsanalyse).

Dem Ziel der Integration in den realen Lehr- und Lernalltag dient auch das betreute Monitoring, ein Beobachtungsverfahren, das wir entsprechend mit einem Bezug zur nachhaltigen Nutzung eingesetzt haben. Der Nachhaltigkeit sollen dienen:

- Übersichtlichkeit durch wenige erhobene Größen, die für die konkrete Entwicklungssituation relevant sind,

- zeitnahe, unmittelbare, prozessbegleitende Datenausgabe,

- große Transparenz für alle Beteiligten durch Ausgabe für alle direkt im System,

- Daten zur tatsächlichen Nutzung aller und nicht von einer Stichprobe sowie nicht nur zur subjektiven Zufriedenheit, die nicht notwendig bis zur tatsächlichen Nutzung reicht,

- kontinuierliche Messung über einen größeren Zeitraum, um Wirkungen auch längerfristig zu beobachten.

\section{Betreutes Monitoring in der interdisziplinären Zusummenarbeit}

Die funktionierende interdisziplinäre Zusammenarbeit ist - so zeigen es auch die Projekterfahrungen in WiBA-Net - notwendige Bedingung für die Entwicklung von Online-Lernprogrammen. Ein ähnliches Ergebnis zeigen Urs Gröhbiels Forschungen: Nur über eine funktionierende interdisziplinäre Zusammenarbeit vermeidet man Ergebnisse, „welche in einem Fachbereich brillieren, aber schwerwiegende Defizite in anderen Bereichen aufweisen“ (Gröhbiel 2001, S. 169). Um etwa Hochglanzprodukte zu vermeiden, die niemand nutzt - die bekannten technischen Ruinen -, bedarf es eines interdisziplinären Zusammenwirkens von Autoren, Informatikern, Didaktikern, Lernpsychologen und Webdesignern. Diese muss dazu aber bestimmten Kriterien genügen, besonders bezüglich der Prozess- und Zielvereinbarung sowie der zu entwickelnden gemeinsamen Sprache. Nur so können „unterschiedliche Vorstellungen zur Gestaltung internetgestützter Lehre“, unterschiedliche „Vorgehensweise(n) im Rahmen der Entwicklung“ und die Entwicklung in ,verschiedenen (Fach-) Sprachen“ sinnvoll integriert werden (vgl. Gröhbiel 2001, S. 27).

9 Der tägliche Bericht, den das System BSCW nach der entsprechenden Einstellung per Email verschickt - so war es im Projekt auch üblich stellt den aktuellen Informationsfluss für alle Projektbeteiligten sicher.

10 Z.B. über die Checkliste, die aus dem Lehrpfadcheck entstanden ist: Deren Kenngrößen - wie angestrebte Kompetenz, eingebundener Test usf. - dienten als den Beteiligten bekannte Maßstäbe für unsere pädagogische Redigierung einzelner Kurse. 
Die interdisziplinäre Zusammenarbeit erfolgte im Projekt WiBA-Net bezüglich der prozessbegleitenden Evaluation und Systementwicklung iterativ. Iterativ meint einen projektbegleitenden, kreisförmigen Prozess, in dem die Projektpartner abwechselnd und rückbezüglich arbeiten: Nach vorgegebenen oder ausgehandelten Kriterien erstellen die Bauingenieure erste Lehrpfade, die die Informatiker in das technische System integrieren. Die ersten Lehrpfade evaluieren die Pädagogen, wobei die Ergebnisse in die Gestaltung weiterer Lehrpfade durch die Bauingenieure einfließen. Ebenso werden bei jedem Durchlaufen des Kreises die Anforderungen an die Technik und Evaluationsdimensionen nötigenfalls angepasst oder neu generiert. Die Rahmenbedingungen für dieses iterative Vorgehen hängen u.a. von den Erfahrungen mit Evaluation und technischem Support ab, von den Erwartungen an die Evaluation und an die technische Unterstützung sowie von den Erwartungen an die Form interdisziplinärer Zusammenarbeit.

Das Monitoring passt gut zu einem iterativen Vorgehen, das angemessen nur interdisziplinär durchgeführt werden kann und zugleich integrierend in den Alltag der Nutzenden wirken soll. Das Monitoring kommt dabei auch der Forderung Urs Gröhbiels bezüglich eines spezifischen „,iterativen Prozesses“ bei der „Entwicklung internetgestützter Lernprogramme“ nach. Iterativ soll der Entwicklungsprozess sein zwischen Bedürfnissen der „Anspruchsgruppen“, z.B. Autoren, Lehrenden und Studierenden, „Lösungsansätze(n)“ und der „Überprüfung der Wirkungen“ (Gröhbiel 2001, S. 168). Nur auf dem iterativen Weg, der diese drei Bedingungen erfüllt, so Urs Gröhbiel, könnten die Lernprogramme überhaupt sinnvoll und nutzerorientiert entwickelt werden. Die Verbesserungen und notwendigen Anpassungen bzw. Veränderungen ergäben sich, indem die Wirkungen, z.B. Nutzungszahlen und Nutzungsverhalten, in diesem Prozess „kontinuierlich verbessert werden“ (vgl. ebd.).

Aber auch die iterative Wirkungsverbesserung funktioniert nicht ohne den frühzeitigen realitätsnahen Nutzerbezug, d.h. das Einbeziehen der spezifischen Wirkung nicht nur im Labor, sondern auch und besonders im vorgesehenen realen Nutzungsumfeld. Ein iteratives Vorgehen erzwingt für die Evaluation, wenn sie auf die Entwicklung und deren Wirkung zielt, ein formatives, d.h. prozesssteuerndes, mindestens aber ein prozessbegleitendes Design, etwa in beratender Funktion. Eine summative Evaluation könnte den Anforderungen an die Entwicklung internetgestützter Lehre im Punkt Iteration nicht gerecht werden. Das Monitoring im Rahmen einer prozessbegleitenden Evaluation ist danach als Methode der Entwicklung von Online-Lernsystemen in interdisziplinären Teams besonders angemessen.

Die Zusammenarbeit zwischen den Projektpartnern wird in disziplinüber- greifenden Kooperationen u.a. problematisch, wenn sich die Projektpartner durch die Evaluation kontrolliert statt beraten fühlen oder wenn sie glauben, in ihrer fachlichen Kompetenz in Frage gestellt zu sein: „Ich lasse mir doch von einem Pädagogen nicht sagen, wie ich einen Lehrpfad zu gestalten habe“; „Warum machen das die Pädagogen? Dann kann ich den Lehrpfad auch meiner 
Frau vorsetzen“ (Originalzitate). Oder sinngemäß formuliert: „Wir nehmen ausschließlich von fachlich kompetenter Seite Kritik am Inhalt an.“

Der letzte Punkt macht deutlich, dass oft nur eine bestimmte Form interdisziplinärer Zusammenarbeit - Experten mit ihren Fachkompetenzen addieren sich - gewünscht ist, die die Disziplingrenzen für die einzelnen Fächer kaum überschreitet. Pädagogen sollen - so die Erwartung - ausschließlich ihre Expertise hinsichtlich der Vermittlung einbringen. Wollen sie jedoch eine Änderung von Inhalten oder Funktionalitäten bewirken, so geht dies, wenn überhaupt, nur vorsichtig und nur unter Bezugnahme auf den Vermittlungsaspekt. Es gibt auch Ausnahmen, aber die sind nicht vom Fach oder der im Projekt üblichen Zusammenarbeit zwischen den Fächern abhängig, sondern von der Einstellung einzelner Personen.

Das betreute Monitoring, als ein Methodenbeispiel aus der Evaluation, soll auch die Besonderheiten der begrenzten, aber durchaus leistungsfähigen interdisziplinären Zusammenarbeit aufzeigen. ${ }^{11}$ Begrenzt meint in diesem Fall eine additive Zusammenarbeit, bei der sich die Disziplinen nur innerhalb ihrer jeweiligen Fachgrenze bewegen.

\subsection{Konzeption: Bedürfnisse und theoretische Einbettung}

Eine von einem Autor angeregte Logfileanalyse ergab eine große Menge an Daten, weil jede Seite einzeln aufgelistet war. Eine Auswertung wäre nur mit großem Aufwand und nur exemplarisch möglich gewesen. Die mit der Evaluation betrauten Pädagogen haben die Anregung zu einer quantitativen Erhebung der tatsächlichen Nutzung allerdings modifiziert aufgenommen und die Methode des betreuten Monitoring konzipiert. Das Monitoring kam den Bedürfnissen der beteiligten Projektpartner - Autoren, Techniker, Projektleitung und Pädagogen - entgegen. Die Partner wünschten sich messbare Daten, die repräsentativ sein sollten. Die qualitativen Erhebungen aus dem Lehrpfadcheck empfanden sie in diesen beiden Hinsichten als ungenügend, weil zu wenige Studierende befragt wurden und dies zu wenig messbare Größen, d.h. in ihren Augen keine Objektivität ergeben habe.

Diese Bedürfnisse wurden aufgenommen und mit den pädagogischen Interessen an die Evaluation verknüpft. Die reale Nutzung sollte der erwünschten gegenübergestellt werden, um eine Nutzungs- bzw. Wirkungskontrolle zu erhalten. Außerdem galt es, die Prozessbegleitung wirksam zu realisieren und sie auch gegen bestehende Vorurteile wirken zu lassen - etwa, dass die Vollständig-

11 Diese Beschreibung ist weiterhin orientiert an den disziplinären Projektaufgaben. Die Interdisziplinäre Zusammenarbeit wäre außerdem noch problemorientiert zu thematisieren: Wie geht man mit auftretenden Problemen um, z.B. mit differierenden Zielen von Disziplinen in der gemeinsamen Arbeit, wenn sie in Konflikt geraten? Entwicklung contra Forschung, Lernen contra Lehren, Lernen verbessern contra Innovation, Mehrwert durch Augmented Learning oder Blended bzw. E-Learning usf. 
keit der Inhalte notwendige und hinreichende Bedingung für die nachhaltige Nutzung sei. Schließlich sollte die didaktisch sinnvolle reale Nutzung im Vordergrund stehen - nicht die Technik - und es sollte ein schnelles Feedback möglich sein. Das nennt, ergänzend zu den Vorteilen bezüglich der Nachhaltigkeit, die positiven konzeptionellen Gründe für die Auswahl der Methode Monitoring sowie der (wenigen) erfassten, ausgegebenen und ausgewerteten Kriterien: „Anmeldung ins System“, „Titel und Anzahl der Kurse“ insgesamt und nach Standorten getrennt.

Das eingesetzte Monitoring erfasste nicht, z.B. über eine detailliertere Logfile-Analyse, die Navigation im System, die Nutzung der Suchfunktion oder die Nutzung anderer Funktionalitäten (Chat, Forum) und Inhalte der Lehrpfade. Die Gründe dafür sind vielfältig. Sie ergeben sich aus Grenzen in der Entwicklungsphase:

- Die reale Nutzung war in den anderen Bereichen in der Konzeptionsphase noch zu gering - Chat, Forum usf. Das ergaben die Interviews mit den Studierenden.

- Inhalte waren noch nicht verfügbar (Infopool, Übungspool, Suchfunktion) oder nur ergänzend in traditioneller Form (ältere Vordiplomsklausuren).

- Die Aufbereitung der Inhalte mit Metadaten und einer nutzbaren Ergebnisanzeige war noch wenig fortgeschritten, trotz bestehender Funktionalität (Suchfunktion).

Für die pädagogische Arbeit, die als prozessbegleitende Evaluation unmittelbar dem Projekt diente und nur mittelbar Forschungsfragen dienen konnte, waren diese Dimensionen zum Erhebungszeitpunkt daher irrelevant.

Auch der notwendig ressourcenschonende Umgang mit technischem Support und eigenen Kapazitäten bezüglich Erhebung und Auswertung machte dieses Vorgehen nötig. Schließlich sind die notwendigen Dienstleistungen von den Projektpartnern aus der Informatik in den Forschungsprojekten noch immer nicht konstitutiv vorgesehen. Der technische Support gilt bisher unmittelbar der Inhaltserstellung. Man war also auf den guten Willen aller Beteiligten angewiesen. Außerdem war der pädagogische Anteil am Projekt mit einer von sechzehn Stellen sehr klein. Die Pädagogen haben daher versucht, die zusätzliche Arbeit für die Kollegen möglichst klein zu halten, die eigene verfügbare Arbeitszeit für die Auswertung realistisch einzuschätzen und nur das zu erheben, was absehbar für Beobachtung und Empfehlungen auch nutzbar und für die Beteiligten hilfreich auszuwerten und zu berichten war.

\subsection{Durchführung: Betreutes Monitoring als Verfahren}

Das betreute Monitoring im Projekt WiBA-Net besteht aus einer computer- und internetunterstützten Datenerhebung der Nutzung eines Online-Systems, die den realen Prozessen und Kontexten vor Ort an den sechs beteiligten Universitäten gegengelesen und in das interdisziplinäre Entwicklungsteam zurück- 
gemeldet wird. Die Rückmeldung der Erhebungsdaten erfolgt unmittelbar. Sie wird als Statistik für alle Entwickler, Lehrenden und Evaluierenden im Lernsystem unmittelbar angezeigt. Die Pädagogen lesen die Daten wöchentlich aus und melden sie in Form einer Kurzauswertung zurück. Die Daten werden durch Nachfragen zu besonderen Nutzungszunahmen bei den Projektpartnern des Bauingenieurwesens vor Ort ergänzt. Die Rückmeldung mit Empfehlungen für Maßnahmen zur Nutzungsförderung des OnlineSystems an den Universitäten erfolgt für ein Semester an dessen Ende als Nutzungsdiagramm mit Kommentaren zu Nutzungshöhepunkten.

Das Monitoring ist als Verfahren etwa zwischen Logfileanalyse und Nutzungsstatistik anzusiedeln. Die folgende Abbildung zeigt die Datenausgabe für die projektbeteiligten Entwickler, Lehrenden, Evaluierenden und Techniker im Projekt WiBA-Net.

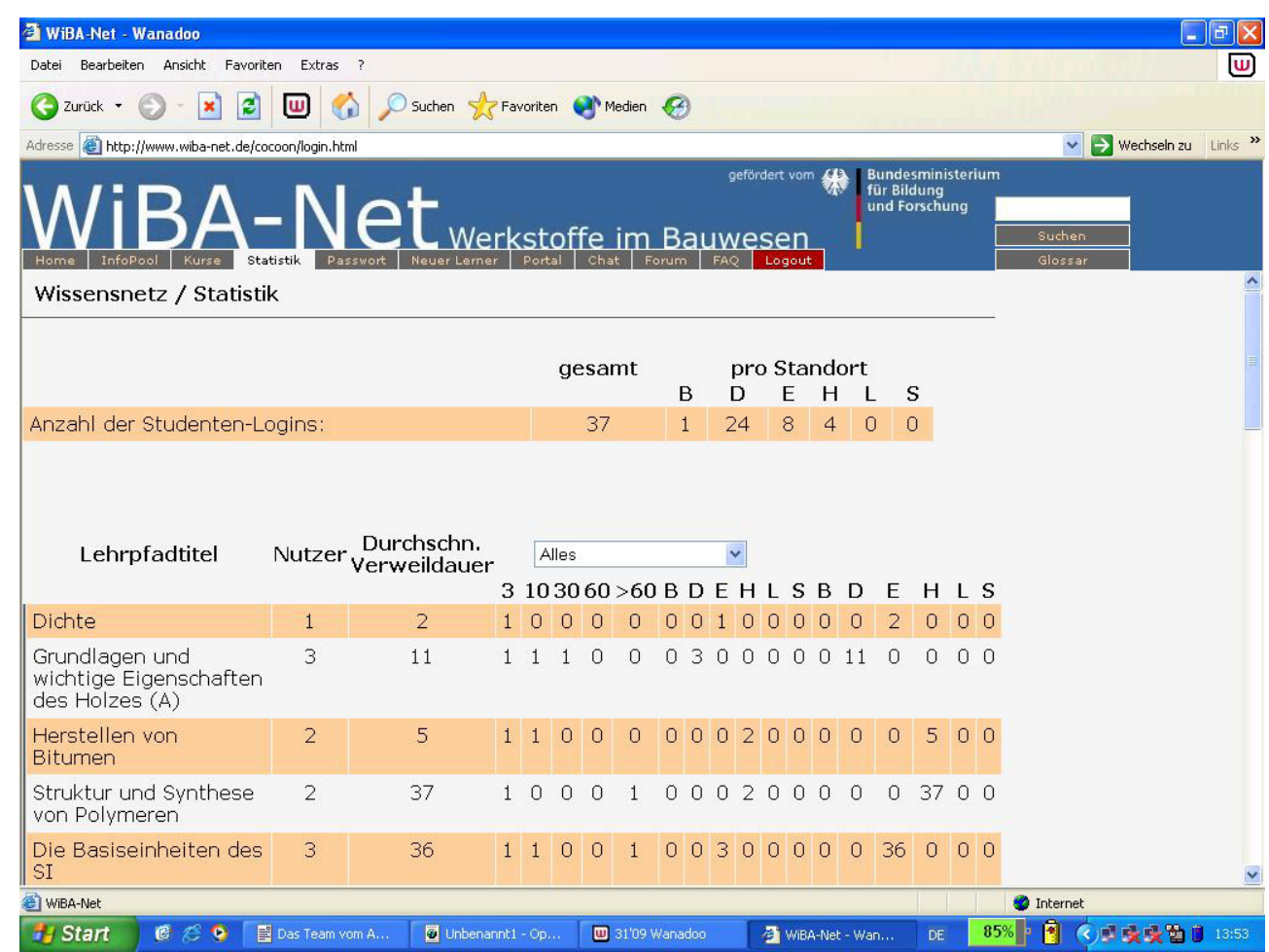

Abb. 1: Datenausgabe für die Projektbeteiligten.

Erfasst wird die Nutzung von Bauingenieurstudierenden in den beteiligten sechs deutschen Universitäten, die das Fach Baustofflehre im Grundstudium studieren und dafür einen Zugang erhalten haben (ca. 500 Accounts). Die Nutzung durch Architekturstudierende war bisher noch unwesentlich. Auf sie entfallen bis heute wenige Accounts und Logins.

Die statistische und nicht personenbezogene Nutzung des Online-Lernsystems pro Woche wird nach folgenden Kriterien erhoben und ausgegeben (vgl. Abbildung 1): 
- Anzahl der Anmeldungen (Logins) ins System ${ }^{12}$,

- Anzahl und Titel der besuchten Lehrpfade,

- durchschnittliche Verweildauer pro Lehrpfad,

- Nutzungszahlen pro Lehrpfad,

- Verweildauer pro Lehrpfad.

Die Daten werden in der Regel für alle Studierenden insgesamt und für jeden der beteiligten sechs Universitätsstandorte einzeln erhoben und ausgegeben. Sichtbar sind sie jederzeit unter dem Button „Statistik“ für alle beteiligten Autoren, Lehrenden, Evaluierenden und Techniker. Die Studierenden wurden auf Wunsch der Autoren zunächst ausgenommen, um eine demotivierende Nichtnutzung in der Entwicklungsphase, so die Vermutungen der Projektpartner, für sie nicht sichtbar werden zu lassen.

\section{Anzahl der Anmeldungen (Logins)}

Im Screenshot haben sich zwischen Montag, dem 29.12.2003 um 0 Uhr und dem Beobachtungszeitpunkt „Donnerstag, 1.1.2004, 14 Uhr“ 37 Studierende aus vier von sechs beteiligten Universitätsfakultäten ins System WiBA-Net eingeloggt. Die meisten Nutzenden, 24, kamen diese Woche bisher aus Darmstadt (D); aus Leipzig (L) und Stuttgart (S) besuchte bisher niemand das System.

\section{Anzahl und Titel der Kurse (Lehrpfade)}

Den Lehrpfad mit dem Titel „Struktur und Synthese von Polymeren“ besuchten z.B. bisher zwei Studierende aus der beteiligten Bauingenieurfakultät in Hamburg (H). Die durchschnittliche Verweildauer betrug bei ihnen und damit auch insgesamt 37 Minuten Dabei gehen eine kurze Nutzung unter drei und eine lange Nutzung über 60 Minuten ein. Die detaillierte Ausgabe der Zeit macht z.B. unangemessene Mittelwerte sofort sichtbar, auch bei größeren Nutzungszahlen.

\section{Betreuung: wöchentlich und semestral}

Die Betreuung fand auf mehreren Ebenen statt. Die wöchentliche Betreuung bestand im Auslesen, Aufbereiten und Auswerten der Daten. Bei bestimmten Ereignissen - wie besonders ansteigender Nutzung - haben die Pädagogen vor Ort nachgefragt, was dort passiert war bzw. was sie unternommen hatten. Diese realen Ereignisse wurden mit den Daten gekoppelt und den Beteiligten sofort als kurze Einschätzung (höchstens eine Seite Text zu den Daten) im Shared Workspace BSCW zurückgemeldet. Der tägliche Bericht, den das System BSCW in der Regel per E-Mail verschickt, stellt den aktuellen Informationsfluss für alle sicher. Damit konnten wir zunächst Hinweise geben. Schon zu Beginn der Beobachtung war z.B. sichtbar, dass die bloße Vergabe von Accounts noch keine Nutzung bewirkte. Eine Erstnutzung fand nur statt, wenn mindestens eine Ein-

12 Mit dem Monitoring erfassen wir zwei der Dimensionen, die Christian Swertz für Online- Evaluationen gefordert hat: Zahl der Anmeldungen ins System und „Start und Ende eines Kurses“, in WiBA-Net Lehrpfad genannt (vgl. Swertz o.J., Abschnitt 2, „Aufzeichnung der Daten“). 
führungsvorlesung, am besten als Online-Präsentation mit typischem Nutzungsweg erfolgte. Schnell sichtbar wurde auch eine Lernquote von zwischen 20 und 30\%: Damit eine Lernnutzung über drei Minuten stattfand ${ }^{13}$ mussten sich im Schnitt zwischen vier und fünf Personen einen Lehrpfad anschauen. Für einen Lehrpfadbesuch bedurfte es zwei Anmeldungen ins System. Somit lernte in den ersten Wochen des Wintersemesters 02/03 etwa jeder achte bis zehnte, der sich in das System eingeloggt hatte.

Das folgende Bild (Abbildung 2) zeigt die Rückmeldung mit Hinweisen konkreter in der Woche XIV im Beobachtungszeitraum WS 02/03:

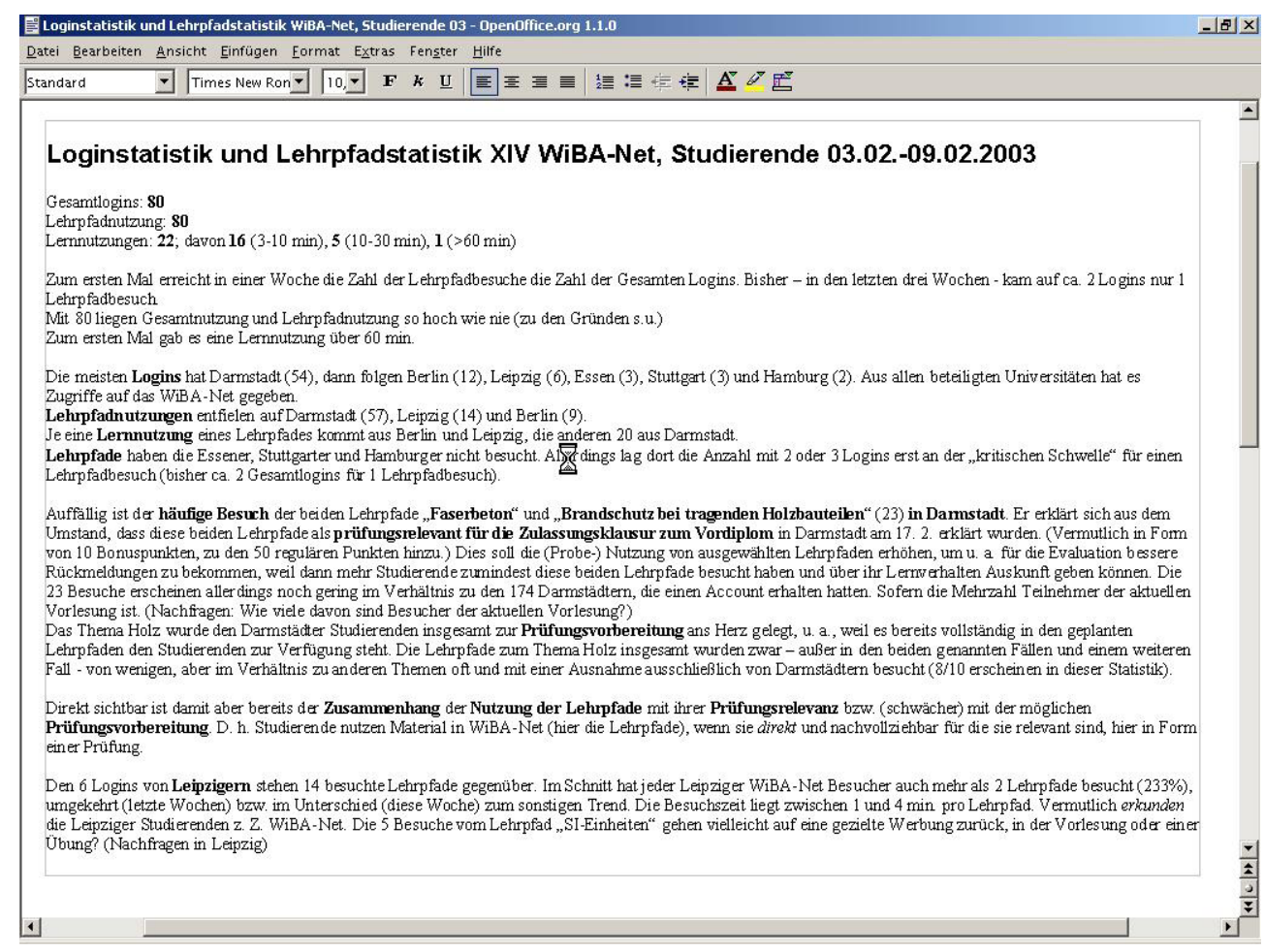

Abb. 2: Wöchentliche Rückmeldung im Beobachtungszeitraum WS 02/03.

Die Hinweise beziehen sich hier auf die Prüfungsrelevanz, die die Nutzung stark und über einige Wochen nachhaltig gefördert hat. Die meisten Vermutungen im Text wurden durch die geplanten Nachfragen vor Ort bestätigt: Es gab eine Einführungsvorlesung mit Online-Präsentation in Leipzig, die erstmals eine Nutzung dort angeregt hatte. Ähnliche Erfahrungen an anderen Standorten führten am Semesterende zu einer entsprechenden Empfehlung. Die Prüfung war nur für etwa 80 von 140 Personen in Darmstadt, die die Vorlesung besuchten, noch relevant. Alle anderen hatten bereits in Prüfungen zuvor oder bei ausländischen Studierenden durch die Anerkennung von Leistungen aus an-

13 Nutzungen über drei Minuten waren Lernnutzungen. Inhalt und Herkunft der Kategorie werden im Rahmen von möglichen Problemen weiter unten diskutiert. 
deren Studienorten die Zulassung zum Vordiplom erlangt. Damit hat im Schnitt jeder, für den die Prüfung relevant war, jeden der beiden Kurse mindestens einmal angeschaut bzw. (offline oder online) mit ihm gelernt. Die Hinweise führten an einem weiteren Standort zu einem ähnlichen Vorgehen bezüglich einer Prüfungseinbindung von Lehrpfaden. Auch dort waren die Ergebnisse sehr gut, besser als im anderen Testteil, so die Rückmeldung auf Anfrage vom zuständigen Mitarbeiter.

Die semesterweise Betreuung erfolgte zudem am Ende eines Beobachtungszeitraums. Am Ende des Semesters entstand eine Grafik, die nach Auffassung des auswertenden Teams die drei wichtigsten beobachteten Größen zeigt.

Weil die Nutzung im ersten beobachteten Semester (WS 02/03) noch gering war, fand zunächst keine Differenzierung nach Universitäten statt:

- Gesamtlogins

- Lehrpfadnutzungen

- Lernnutzungen.

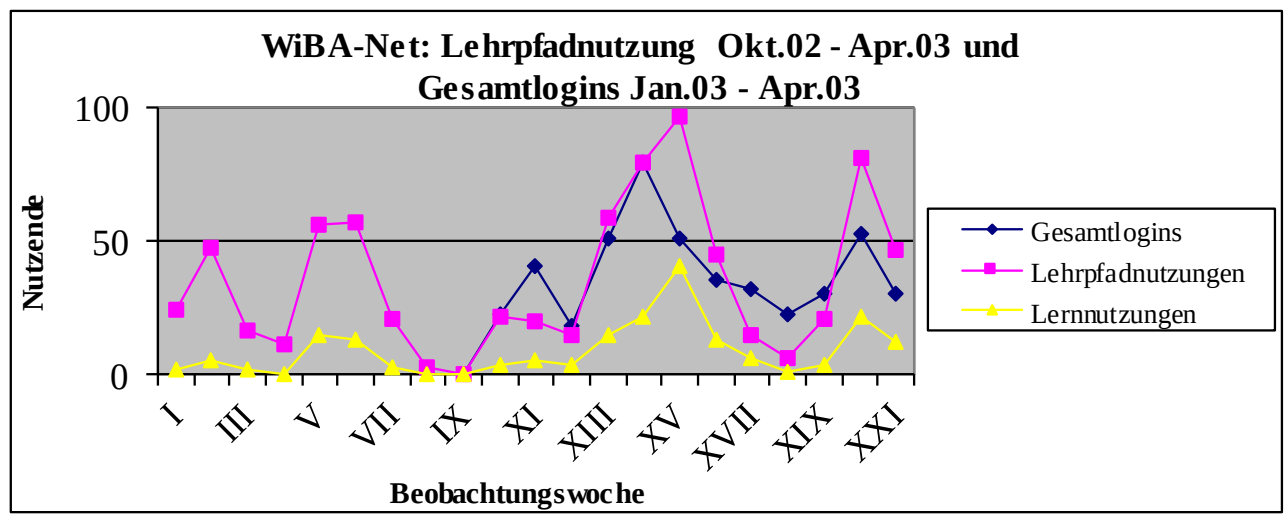

Abb. 3: Logins und Lehrpfadnutzung im WS 02/03.

Auch bei dieser grafisch gestützten Betreuung wurden die realen Ereignisse vor Ort mit den Daten gekoppelt. Die Ergebnisse haben zwei Pädagogen am Ende gesammelt und zusammen mit einer Grafik (Abbildung 3) zurückgemeldet. Das ergab dann nicht nur Hinweise wie in der wöchentlichen Betreuung, sondern auch Empfehlungen, welche Maßnahmen die nachhaltige Nutzung durch Studierende verbessern können. Die Verbindung von Effekten und realen Prozessen in dieser Form ermöglichte den pädagogischen Partnern die Erfüllung einer doppelten Aufgabe, einen praktischen Nutzen im Projekt zu erzielen und zugleich Forschung zu betreiben:

- Die Beteiligten konnten anhand der Evaluation Entwicklungen sehen und erhielten durch die Evaluation Empfehlungen für das weitere Vorgehen und die weitere Gestaltung. 
- Die These der notwendigen Integration in den alltäglichen Lehr- und Lernprozess als notwendiger Bedingung von Nachhaltigkeit konnte so an einem Beispiel geprüft und gefestigt werden.

In der Grafik finden sich vier Nutzungshöhepunkte (Abbildung 3):

a) Der erste Nutzungshöhepunkt (Woche II) konnte sowohl auf eine neugierige Nutzung des Neuen zurückgehen (Hawthorne-Effekt ${ }^{14}$ ) als auch auf die ungewollte Messung der Nutzung von Autoren (fehlende Reliabilität). Da aber beide Effekte nichts bezüglich der Nutzungsförderung aussagen, brauchte diese Frage nicht geklärt zu werden.

b) Der zweite Nutzungshöhepunkt (Wochen V-VI) geht auf gezielte Hinweise auf Lehrpfade in der Vorlesung in Darmstadt zurück. Alle Lernnutzungen und fast alle Lehrpfadnutzungen der Wochen V und VI entfallen auf Darmstadt und auf vier bzw. fünf Lehrpfade zum Thema Beton. Ein Vergleich mit den Folien aus dem Digitalen Hörsaal im Lernsystem (WiBAhö) zeigte, dass Beton in dieser Woche Thema der Vorlesung war. Eine Nachfrage beim Hochschullehrer und seinem Assistenten ergab, dass der Professor in der Vorlesung gezielt auf einzelne Lehrpfade hingewiesen hat.

c) Der dritte Nutzungshöhepunkt (Wochen XIII-XVI) geht auf den Einsatz von zwei Lehrpfaden in einer Zulassungsprüfung zum Vordiplom zurück (Darmstadt). Mit ihnen konnte man Bonuspunkte sammeln (+10 Punkte, zur Punktebasis 50). Den Stoff behandelte weder die Vorlesung noch das Skript. Wer ihn bearbeiten wollte, musste auf WiBA-Net zugreifen.

d) Der letzte Nutzungshöhepunkt (Wochen XX und XXI) ging auf den Einsatz von Animationen in der Sprechstunde an einer Universität (Berlin) zurück: Erklärungen wurden mit Hilfe von Animationen gegeben, veranschaulicht und im Zusammenhang verdeutlicht. Die Studierenden haben dann über das Internet die Animationen und eventuell zugehörige Informationen erneut aufgerufen und nachbereitet.

Besonders aus den Ergebnissen des betreuten Monitoring des Wintersemesters 02/03 ergaben sich eine Reihe von Empfehlungen:

1) Eine Online-Präsentation für die Erstnutzung durchführen, in der das System in spezifischen Nutzungswegen vorgeführt und die Relevanz für das Fach verdeutlicht wird. Nur wenn die direkte Relevanz und der Mehrwert für die Studierenden deutlich wird - Was bringt mir das für meine Prüfung oder meinen Beruf? -, sind viele bereit, den Mehraufwand zu leisten bzw. den Lernweg zumindest ergänzend zu verändern (Online-System statt Skript oder Übungsklausur). Das zeigten die Interviews mit Studierenden.

2) Gezielte Hinweise auf einzelne Lehrpfade - nicht nur allgemein auf das System oder verfügbare Informationen - im Kontext von Vorlesung bzw. Übung für eine stetige, möglichst wöchentliche Nutzung geben.

14 Vgl. zum Hawthorne-Effekt z.B. Schulmeister (1997, S. 397ff., S. 410) oder Kerres/de Witt/Stratmann (2003, S. 1). 
3) Den direkten Einsatz in Prüfungen erwägen. Dem erzwungenen Einsatz ohne Alternative (Vorlesung, Skript, Buch, Übungsklausur), wie im vorliegenden Fall, steht allerdings eine pädagogische Skepsis gegenüber. Die Selbstbestimmung fördert dies nur begrenzt und selten, da die Studierenden nur entscheiden konnten, ob ihnen die normale Vorbereitung genügte - damit war die Prüfung vollständig zu bestehen oder ob sie das zusätzliche Angebot nutzen wollten. Für die erste Alternative entschieden sich (wenig überraschend) aber offenbar nur wenige Studierende (zwei von 30 Interviewten aus Darmstadt antworteten entsprechend). Eine Möglichkeit für ein besseres Prüfungsergebnis nutzen die Studierenden, wenn der Aufwand nicht zu groß ist. Das war der Fall; die zwei ausgewählten Lehrpfade waren angegeben und stofftechnisch überschaubar.

Um aber die Nutzung zu erhöhen und um die Datenerhebung besonders für die Telefoninterviews mit Studierenden zu verbessern bzw. zu ermöglichen, wurde diesem Pilotexperiment dennoch zugestimmt. Sonst hätte die Stichprobe (30 von 120) sicher weniger mit dem System gearbeitet und weniger dazu sagen können.

4) Die Beteiligten anregen, Animationen und Simulationen nicht nur in der Vorlesung und Übung einzusetzen, wie es zunächst geplant war, sondern auch in der Sprechstunde. Die Relevanz wird so direkt im Prozess deutlich. Die Studierenden sehen, dass auch die Lehrenden WiBA-Net offiziell im Lehrendenalltag - hier in der Sprechstunde - nutzen. Die Lehrenden identifizieren sich sichtbar mit WiBA-Net. Die Integration des Lernsystems in den Lehr- und Studienalltag wird dabei soweit gefördert, dass die Studierenden das System mehr nutzen.

Alle beteiligten Partner haben sich sehr positiv über das Verfahren und seine Ergebnisse geäußert. Über die Rückfragen waren alle Beteiligten eingebunden, und sie haben auch mehrfach zu den wöchentlichen Auswertungen Stellung genommen oder Kommentare abgegeben. Für die Techniker war der geringe Aufwand angenehm; interessant sind für sie die realen Nutzungszahlen des Systems. Für uns standen der Evaluations- und der Forschungsaspekt im Vordergrund. Für die Unterstützung der Entwicklung und Evaluation in unserem prozessbegleitenden Ansatz, der auf nachhaltige Nutzung zielt, war das Monitoring sehr hilfreich: Es ist sinnvoll, das unvollständige Produkt bereits in der Entwicklungsphase eines Projektes auszuprobieren und nicht auf das Endprodukt zu warten. Nutzung und Integration sind bereits jetzt wichtige Faktoren, die sinnvoll zu evaluieren sind, neben anderen Faktoren wie der Qualität der Inhalte, der Vollständigkeit und der Funktionssicherheit des Systems.

\section{Probleme der Methode}

Einige mögliche Probleme der Methode seien ebenfalls benannt:

- Validität: Kategorie „Lernnutzung“

- Reliabilität: Datensicherheit

- Effektivität: Verhältnis von Aufwand und Ertrag 
- Flexibilität: Zahl der erhobenen Größen

- Anonymität: Mögliche Überwachungsfunktion des Monitoring.

Nutzungen über drei Minuten waren im vorliegenden Fall Lernnutzungen. Diese Zuordnung ergab sich aus verschiedenen Annahmen und Einschätzungen: In drei Minuten kann der Studierende mindestens eine Seite eines Kurses bearbeiten, so eine orientierende Annahme im Projekt. Sie diente den Autoren neben dem Grundsatz „Eine Seite - ein Inhalt“ als orientierendes Limit für die Gestaltung einer Kursseite. Im Videoprotokoll des Bildschirms besuchte die Probandin in drei Minuten zwischen einer und vier Seiten. Danach absolvierte sie erfolgreich, z.T. mit dem kurzen Rückgriff auf Kursseiten, den Test zum Kurs: Sie hatte also, wenn man - wie hier - entsprechende Vorkenntnisse ausschließen kann, mit dem System gelernt. Außerdem haben einige Studierende die Lehrpfad-Seiten in Word kopiert („Copy \& Paste“), um sie offline am Computerbildschirm zu Hause oder auf Papier zu bearbeiten. Nicht jede kurze Nutzung war also keine Lernnutzung. Die Grenze von drei Minuten kennzeichnet die pädagogische Annahme, dass darunter nur ein Anschauen der Seiten, aber kein Lernen mit ihnen stattfindet. Die Kategorie „Lernnutzung“ kann so zwar begrenzt überprüft und angepasst werden. Sie bleibt aber prinzipiell problematisch, besonders für die Validität, da von der Zeit im Kurs auf das Lernen geschlossen wird. Haben wir das gemessen, was wir messen wollten, Lernen oder doch nur die Zeit oder etwas anderes? Andere Bedingungen und Prozesse können die Zeit stark beeinflussen (Flatrate, Seiten herunterladen). Sie sind außerdem, wie beim Herunterladen von Seiten, u.U. für das Lernen selbst wesentlich. Triangulation begrenzte dieses Problem in seiner Wirkung, sowohl durch Interviewfragen zum Lernen mit dem System für die Prüfung als auch im Vergleich mit den tatsächlichen Prüfungsergebnissen. Die Studierenden hatten mit dem System gelernt, so ihre eigenen Aussagen. Die Prüfungsergebnisse bestätigen diese Aussagen. Die Ergebnisse waren in den betreffenden Aufgaben sogar etwas besser als im restlichen Test. Die Gruppe der Interviewten, 20\% der Vorlesungsteilnehmenden und ca. 35\% der Prüfungsteilnehmenden am Standort der Prüfung war bezüglich des Prüfungsergebnisses repräsentativ. Der Notendurchschnitt der Gruppen war fast identisch, die Notenverteilung ähnlich.

Hinzu kommt das Problem der Datensicherheit. Sie ist problematisch für die Reliabilität, d.h. die Zuverlässigkeit der Daten. Zu Beginn der Erhebung, das deutete der Text bereits an, wurden nicht nur - wie gewünscht - Studierende, sondern vielleicht auch einige Autoren gemessen. Das Problem kann man etwa mit einer getrennten Accountvergabe, z.B. für Gäste, zwar lösen, aber es bedeutet zu einem frühen Projektzeitpunkt, selbst wenn dies vorgesehen ist, eventuell einen zusätzlichen Aufwand für die Techniker. Ein weiteres Problem stellen verlorene Daten auf dem Server dar. Eine tägliche Sicherung mit getrennter Speicherung oder Überschreiben nur bei größeren Zahlen kann hier zwar eine gewisse Abhilfe schaffen, aber auch diese bedeutet einen technischen Mehraufwand. Für die Hinweise auf Ereignisse war dies unkritisch. Andere Verfahren 
(Nachfragen, Interviews) haben zudem die Nutzungszahlen konkretisiert. Ob 15 oder 20 einen Kurs besuchen, ist z.B. für eine quantitative Aussage oder Fehlerrechnung wesentlich. Auch ein Datenverlust in einer Woche ist dann problematisch. Für die verursachende Aktivität und Maßnahmen vor Ort ist die Datensicherheit in diesen Punkten nicht entscheidend, wenn der Fehler nicht sehr hoch ist. Die Interviews und die Prüfungsergebnisse stellten dies sicher (Triangulation).

Mit einer höheren Datensicherheit steigt auch der Aufwand für die Methode (Entwicklung), ebenso durch mehr erhobene Größen (Entwicklung und Betreuung). Dann stellt sich die Frage nach dem Verhältnis von Aufwand und Ertrag sowie nach der ausreichenden Flexibilität der Methode bei steigender Zahl erhobener Größen. Ein Problem besteht schließlich in der möglichen Überwachung durch das Monitoring: Ein Lehrender kann z.B. prüfen, inwieweit seinen Lernempfehlungen gefolgt wird. Bei einer autoritären Lehrperson, die mangelnde Nutzung rügt, kann eine Empfehlung danach u.U. zum problematischen Zwang werden. Wenn die Lerngruppen klein sind, ist eventuell sogar eine Zuordnung von Nutzungen zu Personen möglich. Damit wäre der Datenschutz einer nicht personengebundenen Datenerhebung nicht mehr gewährleistet. Auch in der Weiterbildung, wo der Kontrollaspekt aus ökonomischen Überlegungen leicht im Vordergrund steht, kann dies sicher problematisch sein. Im Kontext der Vorlesungen an der Hochschule vor jeweils 60 bis 140 Personen traten die Probleme des unangenehmen Zwangs (so die Interviewten) und der Kontrolle kleiner Gruppen aber nicht auf.

\section{Forschungsfrage: Online-Lernen und Kursumfang}

Das Monitoring ergab Forschungsergebnisse zur Integration eines Online-Lernsystems in den Alltag als Bedingung von Nachhaltigkeit, zur Prüfung als einem Hauptmotiv der Nutzung und zu didaktischen Empfehlungen. Es erbrachte zusätzlich Daten zum OnlineLernen in Abhängigkeit vom Kursumfang. Die in einem Test in Darmstadt eingesetzten zwei Lehrpfade differierten stark im Umfang. Ein Lehrpfad zum Thema Holz umfasste fünf Seiten, der andere zum Thema Beton 25 Seiten.

Betrachtet man den Besuch über die vier Wochen bis hin zur Prüfung (Wochen XIIIXVI des Beobachtungszeitraumes im WS 02/03), dann ergeben sich für beide Kurse ähnliche Nutzungszahlen, insgesamt 76 bzw. 68. Man sieht diesen Zahlen nicht direkt an, ob wenige Nutzende die Kurse oft angesehen haben oder viele nur einmal. Die Interviews zeigten aber, dass fast alle die Kurse kannten und für die Prüfung auch bearbeitet hatten. In der Tendenz haben viele Nutzende die Kurse jeweils einmal angeschaut.

Alle Nutzungen entfallen auf den einen Standort der anstehenden Prüfung. Die zeitliche Verteilung bezieht sich auf die vier Wochen vor dem Test (XIII-XVI), wobei alle Nutzungen der letzten Woche nur am ersten Tag, dem Montag der Prüfung, stattfanden. 


\begin{tabular}{llllllll}
\hline Lehrpfadtitel & $\begin{array}{l}\text { Nutzungen/ } \\
\text { Wochen } \\
\text { XIII-XVI }\end{array}$ & $\varnothing$-Zeit & $<3$ min & 10 min & 30 min & 60 min & $>60$ min \\
\hline $\begin{array}{l}\text { Brandschutz bei } \\
\text { tragenden }\end{array}$ & 76 & 8 & 44 & 14 & 17 & 1 & 0 \\
$\begin{array}{l}\text { Holzbauteilen } \\
\text { Faserbeton }\end{array}$ & 68 & 16 & $\begin{array}{l}(58 \%) \\
(59 \%)\end{array}$ & $\begin{array}{l}(18 \%) \\
(15 \%)\end{array}$ & $\begin{array}{l}(22 \%) \\
(18 \%)\end{array}$ & $\begin{array}{l}(1 \%) \\
(6 \%)\end{array}$ & $(4 \%)$ \\
\hline
\end{tabular}

Tab. 1.: Nutzungsdauer in den vier Wochen bis zum Test.

Die Durchschnittszeiten ergeben prinzipiell erwartete Werte. Im kurzen Kurs liegt die Besuchszeit pro Kurs im Schnitt bei acht Minuten, im langen Kurs bei doppelt so langen 16 Minuten. Die Verteilung zeigt aber, dass fast $60 \%$ der Nutzungen in beiden Fällen unter drei Minuten liegen (vgl. Tabelle 1). Der Mittelwert sagt im vorliegenden Fall also zu wenig aus.

Bei etwa gleichen Nutzungszahlen differiert die aufgewendete Zeit in der Zeitverteilung kaum, obwohl der eine fünf und der andere 25 Seiten umfasst. Nur bei den langen Nutzungen über 30 Minuten gibt es beim langen Kurs erwartungsgemäß mehr Nutzungen, aber nur einige (10\% gegenüber $1 \%$, vgl. Tabelle 1). Bei den mittleren Nutzungszeiten (drei - 30 Minuten) gibt es beim kürzeren Kurs im Verhältnis etwas mehr Nutzungen (40\% gegenüber 33\%). Eindeutig überwiegt aber bei beiden Kursen die kurze Nutzung unter drei Minuten. Die Zeitverteilung der online für einen Kurs verwendeten Zeit bleibt überraschend für die Kurse insgesamt ähnlich. Etwas anders sieht es aus, wenn man nur die letzte vollständige Woche vor dem Test betrachtet (XV). Die folgende Darstellung zeigt die Nutzung:

\begin{tabular}{llllllll}
\hline Lehrpfadtitel & $\begin{array}{l}\text { Nutzungen/ } \\
\text { Wochen } \\
\text { XIII-XVI }\end{array}$ & $\varnothing$-Zeit & $<3$ min & 10 min & 30 min & 60 min & $>60$ min \\
\hline $\begin{array}{l}\text { Brandschutz bei } \\
\text { tragenden }\end{array}$ & 23 & 12 & 8 & 4 & 10 & 1 & 0 \\
$\begin{array}{l}\text { Holzbauteilen } \\
\text { Faserbeton }\end{array}$ & 46 & 11 & $\begin{array}{l}32 \\
(70 \%)\end{array}$ & $\begin{array}{l}3 \\
(18 \%)\end{array}$ & $(43 \%)$ & $(4 \%)$ & $(-)$ \\
& & & $(70)$ & $(15 \%)$ & $(4 \%)$ & $(4 \%)$ \\
\hline
\end{tabular}

Tab. 2.: Nutzungsdauer in der Woche vor dem Test.

Nur in der Woche vor der Prüfung, wo es unmittelbar um das Lernen für die Prüfung geht, haben fast zwei Drittel der Studierenden, die die beiden Kurse besuchten, den Kurs mit fünf Seiten, für den ca. 10 bis 15 Minuten Lernzeit veranschlagt waren, tatsächlich am Bildschirm online gelernt. (So auch die Bestätigung durch die Telefoninterviews.) Die wesentliche Nutzungszeit des Lehrpfades „Brandschutz bei tragenden Holzbauteilen“ liegt über 3 Minuten (15/23 =65\%, vgl. Tabelle 2) und zwischen 3 und 30 Minuten (61\%). Die im Projekt 
angestrebte Lernzeit pro Lehrpfad von höchstens 30 Minuten scheint sich hier als gute Wahl zu bestätigen, wenn man eine Online-Nutzung anstrebt.

Das Anschauen bzw. die Vorbereitung mit dem langen Kurs „Faserbeton“ haben $70 \%$ auf die letzte Woche verschoben. Den kurzen Kurs hatten sie sich auch schon in den Wochen davor zumindest angesehen. Die wenigen, die den langen Kurs online bearbeitet haben, taten dies vorwiegend schon zwei Wochen vor dem Test (8 von 9, o. Abbildung). In der letzten Woche haben ihn zwar auch noch 14 von 46 länger als drei Minuten bearbeitet, aber für die meisten reichte es nur noch zum Anschauen oder Herunterladen von Seiten (32 von 46, vgl. Tabelle 2). Dies gaben drei Befragte explizit in den Telefoninterviews an.

Das wegen geringer Nutzungszahlen nur exemplarische und hinweisende Ergebnis ergibt weiteren Forschungsbedarf bzw. mögliche Forschungsfragen. Hat die Seitenzahl tatsächlich kaum einen Einfluss auf die Bearbeitungszeit online? Woran liegt das? Welchen Einfluss haben hier die Präsentationsformen? Legt der in den beiden Kursen vorherrschende Text mit Bildern eher das Herunterladen nahe? Was beeinflusst dann die tatsächliche Bearbeitungszeit (nachhaltig), wenn die Seitenzahl kaum einen Einfluss hat? Stellen z.B. die Kosten für den Internetzugriff von zu Hause aus eine mögliche oder gar die entscheidende Größe für die Bearbeitungszeit dar? „Zu Hause“ bezeichnet eindeutig den vorwiegenden Ort und den Wunschort der studentischen Nutzung von WiBA-Net, so die Telefoninterviews. Den Punkt „Kosten“ benannten in den Telefoninterviews nur wenige Studierende in einer offenen Frage zu Nutzungshindernissen als für sie relevante Nutzungsbedingung. Der Aspekt wurde aber nicht explizit bei allen erhoben.

Von den Nutzungen unter drei Minuten müssen viele dennoch als Lernnutzungen gewertet werden. Die zugrunde gelegte Kategorie „Lernnutzung“ von drei Minuten muss danach - wie angedeutet - neu bewertet werden. Die Studierenden haben sich zum Teil, das ergaben mündliche Rückmeldungen vom Assistenten und die Telefoninterviews, Lehrpfadseiten in Worddateien kopiert, um sie anschließend auszudrucken. Mit diesem Ausdruck haben sie dann wie üblich gelernt, wie z.B. mit dem Skript. Für diese Gruppe spielt die Lehrpfadlänge in der letzten Woche der Prüfungsvorbereitung eine Rolle beim Bearbeitungsmodus: Den kurzen Kurs haben sie online bearbeitet, den langen aus zeitökonomischen Gründen heruntergeladen und auch nur Ausgewähltes gelernt.

Der genaue Anteil ist allerdings nicht auszumachen, da man den Zahlen nicht ansehen kann, ob sich hinter ihnen „bloß rein gesehen“ oder „Seiten heruntergeladen“ verbirgt. Zumal die unvorhergesehene Nutzung des Seiten-Herunterladens mit zunehmenden Multimediaanteilen schwieriger bis unmöglich wird: Wie soll ich eine Animation oder Simulation seitenweise herunterladen und wie sinnvoll ist dies? Der Multimedianutzung kommt in jedem Fall die Online-Nutzung eher entgegen. Allerdings können die Autoren und Betreiber eines solchen Lernnetzwerks überlegen, ob sie Versionen der Lehrpfade zum Ausdrucken verfügbar machen möchten, wie es einige Studierende wünschen, um ihnen so weit wie möglich eine Nutzung nach ihren Vorstellungen zu er- 
möglichen. Oder ob sie das nicht wollen, weil es nicht die Stärken des Mediums - Multimedia - nutzt bzw. fördert, sondern im Gegenteil eher die traditionellen Wege. Diese Frage wurde mit diesen Überlegungen an die Autoren und Betreiber zurückgegeben, damit sie die Entscheidungsnotwendigkeit erkennen und die Frage für sich entscheiden.

\section{Fazit: Betreutes Monitoring}

Das betreute Monitoring ist eine Methode, die in der Entwicklung, Evaluation, Erforschung und Durchführung von Online-Lernen und Blended Learning sinnvoll einsetzbar ist. Sie kann Hinweise liefern und Empfehlungen zur Nutzungsförderung generieren. Sie kommt den Anforderungen, die interdisziplinäre Entwicklungsgruppen mit sich bringen, bereits mit begrenztem Aufwand entgegen. Für umfangreichere und direkt quantitativ reliable und valide Ergebnisse (genau messen und sicher messen, was man messen möchte), müsste sie entsprechend ausgebaut und angepasst werden. Probleme ergeben sich dann, aber abgestuft auch allgemein, nicht nur für die Zuverlässigkeit der Messergebnisse (Datensicherheit), sondern auch für das Verhältnis von Aufwand und Ertrag sowie bei kleinen Gruppen und bestimmten Kontexten eventuell bezüglich des Datenschutzes und der Kontrollmöglichkeit.

In der begrenzten Form, die hier vorgestellt wurde, kommt das Monitoring iterativen Entwicklungs- und Evaluationsprozessen im Bereich Blended Learning und E-Learning besonders entgegen, die kleine, flexible sowie schnell sichtbare und wirksame Ergebnisse einschließlich Feedback benötigen. Diese Anforderung an kleine, anforderungs- und situationsspezifischer einsetzbare Methoden wird umso bedeutsamer, je umfangreicher der iterative Prozess und je größer das angestrebte Produkt ist.

Bereits mit dem Erfassen, Ausgeben und Auswerten der Anmeldungen ins System (Logins) sind erste, relativ einfach zu erhebende Daten zur tatsächlichen Nutzung des Online-Systems zu gewinnen. In der Verbindung mit dem Erheben von Zugriffen auf Lehrpfade (Titel, Anzahl, Dauer) kann die Nutzung und Akzeptanz des Systems und der Inhalte im Verhältnis dazu sichtbar gemacht werden. Über andere Methoden wie Interviews und Fragebögen sind Rückkopplungen und Vertiefungen in Entwicklung, Evaluation und Forschung sinnvoll möglich bzw. nötig, z.B. zur Kategorie „Lernnutzung“ von Online-Inhalten. Auch Detailfragen können untersucht bzw. für die weitere Forschung konkretisiert oder generiert werden. 


\section{Literatur}

Blumstengel, A.: Entwicklung hypermedialer Lernsysteme. Berlin 1998. Als Hypertext in: http:// dsor. uni-paderborn.de/de/forschung/publikationen/blumstengel-d.iss/main_index_netzwerk. html.

Gröhbiel, U.: Entwicklung internetgestützter Lernprogramme. Am Beispiel des Internet-Lehrgangs „AREA“ (Access to Regional Economic Approaches). Dissertation. Basel 2001.

Kerres, M./de Witt, C./Stratmann, J.: E-Learning - Didaktische Konzepte für erfolgreiches Lernen. In: Schwuchow, K.-H./Guttmann, J. (Hrsg.): Jahrbuch Personalentwicklung und Weiterbildung. München 2003. Online unter: [http://edumedia.online-campus.net/publications/jahrb-pe-wb-b.pdf] (30.01.2004).

Mayring, Ph.: Methoden der qualitativen Sozialforschung. Weinheim 2002.

Meuser, M./Nagel, U: Experteninterviews - vielfach erprobt, wenig bedacht. In: Garz, D./Kraimer, K (Hrsg.): Qualitativ empirische Sozialforschung. Konzepte, Methoden, Analysen. Opladen 1991, S. 441-471.

Schulmeister, R: Grundlagen hypermedialer Lernsysteme. Theorie - Didaktik - Design. 2. aktual. Auflage. München 1997.

Sesink, W: Theoriebildung und Projekterfahrung. Ein Bericht. Darmstadt 2002. Online unter: www. sesink.de

Swertz, Ch. (in Druck): Selbstevaluation im Online-Lernen. Erscheint in: Meister, D. (Hrsg.): OnlineLernen und Weiterbildung, Abschnitt 2, „Aufzeichnung der Daten“. Als html-Datei verfügbar unter: www.swertz.de (23.01.2004) 